\title{
Often a Sick Child from the Perspective of a Pediatrican/Clinical Immunologist
}

\author{
Jaromir Bystron \\ Department of Allergy and Clinical Immunology, University Hospital, Ostrava, Czech Rep \\ Email address: \\ jaromir.bystron@tiscali.cz \\ To cite this article: \\ Jaromir Bystron: Often a Sick Child from the Perspective of a Pediatrican/Clinical Immunologist. American Journal of Pediatrics. \\ Vol. 7, No. 2, 2021, pp. 57-61. doi: 10.11648/j.ajp.20210702.14
}

Received: March 22, 2021; Accepted: April 6, 2021; Published: April 13, 2021

\begin{abstract}
The author considers the term "often a sick child" and discusses this topic in relation to the gradually maturing immune system. He points out the specifics of different age periods and tries to prove that the not always higher frequency of diseases (especially the most common ones - respiratory tract infections) must have a cause in the immune system. Above all, it is necessary to distinguish between primary-congenital immune disorders, which often require lifelong specialized care, from secondary-acquired disorders, for which it is most important to identify the cause of such a disorder and try to eliminate it. An irreplaceable role in the diagnosis and care of often ill children is played by general practitioners caring for children and adolescents, who know the environment in which the child develops and have the possibility of basic immunological examination to identify more serious immune disorders.
\end{abstract}

Keywords: Often Sick Child, Immune Disorder, Congenital Immunodeficiency, Acquired Immunodeficiency

\section{Introduction}

The present time, which we like to call "modern times", is characterized by some facts. Our life is very hurried, every hour, minute we have planned for some activity mainly within various duties (work, social, family) and we are less concerned with relaxation, rest, sleep, proper nutrition and every disruptive event that this system breaks down. it bothers us, it can drive us crazy. One such event that can disrupt the time schedule is also the child's illness. Such a disease forces us to interrupt the course of our lives. Since we can't put a child in kindergarten or school, they force us to stay at home with him (unless we have a free grandmother or we can't afford a nanny or nurse), interrupt work, cancel appointments, social gatherings and the like. If it happens once or twice a year, it's still acceptable, it's manageable - it has to be taken into account. But when it happens several times a year, it is a problem and the typical reaction of the parents to the doctor is: "Doctor, the child is often ill somehow, he certainly has an immune disorder", in the worst case: "the child has no immunity." Is it really a matter of immunity or is the problem elsewhere? Parents like to look for a simple answer and also a simple solution: "Doctor, give him/her something to support his immunity, so that he is not so often ill".
The problem in most cases is not a disorder of immunity, it is much more complex and usually more in the family, its lifestyle and habits, which parents do not want to hear much about, because they would have to change this style, habits [1-4]. The situation with an often ill child is different in different age groups, so I will try to approach the specifics of different age groups.

\section{Immunological Specificity of Different Age Periods}

\subsection{Immunity in Infancy and Preschool Age}

Gradually maturing immune system. The dynamics of the immune system's maturation can be very different for each child. A very good indicative indicator is the anamnestic inquiry into morbidity in infants and toddlers with parents or siblings. Information on tolerating vaccinations and information on morbidity after the child's inclusion in a larger group - nursery, kindergarten, entry into primary school - is important.

\subsubsection{Congenital Immunodeficiencies}

Serious immunodeficiencies manifest soon after birth, in 
infancy, at the latest in infancy, serious infections of the respiratory tract, skin, severe diarrhea and must be addressed as soon as possible by sending for examination to a specialized workplace, which is mostly part of the children's ward of a regional or university hospital. Milder forms of immunodeficiency appear mainly after the inclusion of children in larger groups during toddler and preschool age. Their treatment is initially symptomatic, according to the current condition of a general pediatrician. In case of recurrent or disproportionately long and severe ongoing infections, examination is necessary in a specialized clinic of allergology and clinical immunology, which are in in the Czech Republic in every major city (currently there are approximately 3.5 medical positions in the field of allergology and clinical immunology per 100,000 inhabitants).

It is considered a warning sign of a congenital immune disorder 4 or more bacterial infections require ATB treatment within 1 year (otitis, bronchitis, sinusitis, GIT infections).

Two or more serious bacterial infections (pneumonia, meningitis).

Repeated infections or infections requiring prolonged or i.v. treatment of ATB.

Recurrent infections located in different parts of the body

Insufficient increase in the level of protective antibodies after vaccination.

A poorly thriving child for no apparent reason.

Family history of immunodeficiency or recurrent infections.

Extensive persistent candidiasis after 2 years of age $[5,6]$.

\subsubsection{Joins the Children's Team}

The inclusion of a child in the team leads to a significant expansion of contact with droplet air spreading infection, and in children who are not yet fully immunologically mature, this contact can cause more frequent catarrhal manifestations, especially in the upper respiratory tract. Experience shows that this "physiological prominence - physiological immunization" is useful if the individual infections are mild and children are provided with proper recovery. These are mostly (up to $90 \%$ ) viral diseases that do not require antibiotic treatment. Examination and treatment are fully within the competence of general pediatricians. In recurrent patients (more than 6-8 infections per year are clearly relapsed per year, clearly diagnosed and requiring rest, ATB treatment or hospitalization) or severe or prolonged (requiring repeated ATB administration or hospitalization), examination in an allergy and clinical outpatient clinic is indicated. Immunology.

\subsubsection{Insufficient Hardening}

Improper dressing and insufficient physical activity are often the cause of more frequent morbidity in children with upper respiratory catarrh. For children performing physical activities, the clothing (number of layers of clothing) should be the same as for adults performing similar activities. Excessive clothing causes increased sweating, increased fatigue and consequently easier cooling after "blowing" in windy weather while calming physical activities. The principles have always been and still are that for infants who do not move on their own and are passively carried or transported in prams, special carts should have a warm body, and for children moving, their feet, head and neck must be dry and warm.

\subsubsection{Incorrect Diet Composition}

Here I come across the consumption of delicacies, sweets, sugary drinks, which are produced by our industry primarily to induce children's addiction to sweet tastes and colors and do not respect the nutritional needs at all. The high content of sugar or other sweeteners, preservatives and many other artificial chemicals makes children addicted to these "foods" and children do not consume natural nutrients, vitamins, water, minerals from natural diets - fruits, vegetables and the like. This creates "hidden dementia secondary foodborne immunodeficiencies", which we cannot easily and quickly identify in the laboratory, we only suspect and cause obesity, irritability, fatigue and more frequent illness in children by weakening immune functions.

\subsection{Immunity in School Age and Adolescence}

Termination of the basic maturation of the immune system. The "maturation of the immune system and its functions" continues. Non-specific immunity is generally fully mature, but adaptive (acquired) immunity is still maturing due to the environment. The levels of some antibodies (eg immunoglobulins) reach the level of levels that are common in adults in children up to 6-10 years of age. This adaptive immunity creates an "immunological memory" that applies the fact that an individual already responds to an infection he or she has encountered at a pre-school age with a much faster and more effective immune system, so there are fewer "common colds and infections". than in preschool age. In layman's terms, it is well characterized by the sentence Schoolchildren have already grown out of those common infections. In addition, the state health system itself helps to develop children's "immunological memory" through vaccination against serious diseases, which in the past caused widespread epidemics, high morbidity and mortality and are now only sporadically visible due to compulsory vaccination (diphtheria, whooping cough, measles, rubella, mumps, polio, tuberculosis) although, unfortunately, thanks to some "anti-vaccination activities") and often with a serious course.

\section{Examination of Patients with Suspected Immune Disorders}

As with many medical disciplines, a very careful medical history is the basis. In the family history, we look for deaths in the family in infancy or toddler age, questions about birth defects, chronic diseases, serious infections, frequent illness, especially in the patient's parents and siblings. In the personal history we start during pregnancy (important are data on smoking during pregnancy, alcohol and drug consumption), maternal illness during pregnancy, we ask about the course of childbirth, fetal vitality after childbirth, thriving in the first 
days and months, postpartum jaundice, congenital malformations. We carefully determine the frequency and severity of infections in infancy and subsequent toddler age, reactions to vaccination. Important information is data on the child's morbidity after joining the children's group (nursery, kindergarten and then primary school), the course of common infections of childhood. In late school age, adolescence, in addition to questions about the frequency, course and severity of infections, are important information about the family environment, mental resilience in crisis situations, the environment in which the patient lives.

What is the basic and what is the specialized laboratory immunological examination

Laboratory examination of a patient with a suspected immune disorder begins with a routine examination of blood count and differential budget, which we supplement with examination of inflammatory markers (CRP, erythrocyte sedimentation) and examination of basic classes of immunoglobulins (IgM, IgA, IgG, IgE). This basic examination can provide us with important information about the current state of the body - leukocytosis or leukocytopenia, with specifications - lymphocytopenia, monocytosis, eosinophilia, anemia, hypo- or agammaglobulinemia or elevated values of individual classes of immunoglobulins that contribute to the diagnosis of acute or chronic inflammation, allergies or immunodeficiency and may also significantly indicate a specific diagnosis - monocytosis in infectious mononucleosis, atypical forms of blood elements in hemoblastosis, increased eosinophils and total $\mathrm{IgE}$ in allergies or parasitic diseases. All these examinations are also available to general practitioners, have a very good informative value and are not economically demanding.

Basic immunological examination in patients with suspected immune disorders.

1. a detailed family and personal history with a focus on immune disorders.

2. laboratory examination: FW, CRP, KO + diff., IgM, IgA, IgG, IgE.

By specialized immunological laboratory examination we specify the possible disorder by a more detailed examination of individual components - examination of immunoglobulin subclasses IgG (IgG1-4), IgA (IgA1-2), examination of specific IgE for individual allergens, examination of complement components (C3, C4, total complement, $\mathrm{C} 1-\mathrm{INH}$, functional $\mathrm{C} 1-\mathrm{INH})$, examination of phagocytosis, intracellular killing, examination of surface features on lymphocytes and identification of subpopulations of lymphocytes $(\mathrm{CD} 3+, \mathrm{CD} 4+, \mathrm{CD} 16+56+, \mathrm{CD} 19+, \mathrm{CD} 20+$ and others), examination of general and specific signs of autoimmunity (ANA, ANCA, ENA, rheumatic factors, specific factors for organ autoimmunity, examination of functional activity of lymphocytes using lymphotransformation tests, examination of basophil activation, skin tests with inhaled or bacterial allergens and numerous others, which are reserved for specialized clinics of allergology and clinical immunology, rheumatology or some other specializations. Their indication and subsequent interpretation in the practitioner should entrust the results to experienced specialists and not induce large panels of these tests himself [7].

\section{Definition of Recurrent Respiratory Infections}

Recurrent infections, especially of the upper respiratory tract, are a classic manifestation of the above causes. The crucial question is - What is a common illness? In this case, it is a consensual (agreement) term. In various clinical studies, increased morbidity in this age group of children is considered to be the need for ATB treatment more than 6-10 times per year. Unfortunately, the administration of ATB is often overused in our country, when we know that respiratory infections in children at this age are up to $90 \%$ caused by viruses. The administration of ATB in this case is not only ineffective, but also causes a disorder of the natural intestinal bacterial population (intestinal microbiots), which may have a further adverse effect on the child's health development.

The time of regeneration should be strictly observed joining the team only after complete recovery. An untreated child is always more prone to another illness if he has not yet recovered from one illness. In collective facilities, parents should monitor the illness of other children or inquire about it with teaching staff. On the one hand, he finds out "what kind of disease is currently rushing among the children" and also learns whether their child somehow exceeds the average of the collective by the frequency of his illness. Preparations for activating immunity, supporting the maturation of immune functions, whether prescription or over-the-counter, should be consulted with a pediatrician who is familiar with the child and can assess whether the condition is already in need of immunological intervention. Parents usually react emotionally and very subjectively. It is understandable that they want their child not to moan, to be able to go to work on their own, not to take time off to care for the child and not to stay at home with him for a long time in convalescence, but life is about something else. Adequate morbidity of children is completely physiological, it is a natural "training of the immune system".

It is also often a naive notion that such a common illness will be cured when we send a child to a spa. Spa treatment is very effective in specific diseases (conditions after severe pneumonia, in asthmatics, in children with chronic respiratory diseases), but not in common diseases. In such a case, the spa can act as a transition to another kindergarten, where the child meets other "more often ill children", and thus with another type of mostly viral infection and the infection in the spa also gets sick and parents then complain that their child half time in the spa was sick. It is much better to use such climatic treatment (mountains, sea) as part of an extended family vacation.

In children 2-8 years: at least 3 infections in the last 3 months or 4 in 6 months or 6 per year.

In children 8 years and older: at least 2 infections in the last 3 months or 3 in the last 6 months or 4 per year. 
An infection is considered to be a condition requiring at least a 3-day rest period, dropping out of school or using antibiotics and meeting at least 3 of the following objective criteria. - more than 3 days of subfebrile illness or temperature rise above 38 st at least one day, acute rhinitis, sudden cough, sore throat, headache, otalgia, exacerbation of fatigue with the possibility of closing the diagnosis - rhinopharyngitis, pharyngitis, laryngitis, tracheitis, bronchitis, pneumonia, sinusitis, otitis, tonsillitis apod.

\section{Principles of Treatment of Recurrent Infections}

1. Thorough examination and treatment of the basic cause of the current disease - substitution of body fluids, proteins, minerals, vitamins, targeted use of antibiotics, antivirals, antifungals according to the current state and sensitivity.

2. Careful search for foci of infection (paranasal sinuses, middle ear, odontogenic infections, urinary tract, small subcutaneous abscesses, often adenoid vegetation in children, etc.) and their proper treatment.

3. Adequate reconvalescence, regimen and dietary measures after the measures referred to in points 1 and 2 .

4. Use of immunostimulatory or immunomodulatory agents with insufficient success in the previous three points.

Treatment must then be focused on the identified primary cause, proper convalescence, and in case of insufficient effect of these basic procedures, the right treatment includes the right timed and correctly chosen immunomodulatory treatment.

In cases where a specific immune disorder is identified as the cause of recurrent RI infections, immunosubstitution therapy is appropriate. Where the immune response is manifested by enhanced defensive inflammation, we use anti-inflammatory therapy and, conversely, where we need to activate some transiently weakened immune mechanisms, we use immunostimulatory agents. Immunostimulatory agents can be used only where the immune system is formed in its individual components, that is, we can stimulate only something that is present in the body, but for certain reasons (exhaustion, starvation, chronic infection, extensive trauma, immunosuppressive therapy and the like) is temporarily insufficiently effective.

Does a sick child often have a general practitioner or specialist treated for recurrent infections?

If congenital immunodeficiency is suspected - frequent infections from birth (pneumonia, otitis, sinusitis, skin infections, more severe gastrointestinal tract infections, severe vaccination reactions) or more severe secondary immunodeficiency (eg conditions after major surgery, immunosuppressive or radiation therapy) followed by increased frequency and severity of infections, a more detailed immunological examination is clearly indicated and immunomodulatory treatment is then conducted according to the results of this examination and under the supervision of a specialist allergist/clinical immunologist. However, if the history confirms the very common frequency and severity of infections in childhood and adolescence, and the more frequent morbidity turns out to be a completely new factor, there is no reason why it could not be addressed by a general practitioner. If necessary, even a general practitioner can perform a simple (and inexpensive) basic immunological examination (see above). However, even in these conditions, it is very useful to shed light on the beginning of the deterioration of the health condition with the subsequent development of recurrent respiratory RI infections. It can be a change of residence, children's team, employment and work team with parents, transport to work - in public transport, eating habits, depression in solving personal problems, underestimation of convalescence in the treatment of a more serious infection. If such a cause can be unambiguously identified, it is necessary to address this cause first and then proceed to immunomodulatory treatment [8-12].

\section{Conclusion}

An often sick child - especially with recurrent upper respiratory tract infections - may not always be the result of an immune disorder. However, if an immune disorder is suspected, it is fully within the competence of a general pediatrician with a detailed history, analysis of the family environment and basic immunological examination to try to distinguish between primary-congenital and acquired secondary immunodeficiency. If it is not a serious condition, it is also in his competence to try to treat these problems. In case of a more severe course or if a congenital immune disorder is suspected, a specialized examination in the outpatient clinic of allergology and clinical immunology is fully indicated.

\section{References}

[1] Toivonen L, Karppinen S, Schuez-Havupalo L, Teros-Jaakkola T, Vuononvirta J, Mertsola J, He Q, Waris M, Peltola V: The burden of recurrent respiratory infections in children: A prospective cohort study. Pediatr Infect Dis J. 2016 December; 35 (12): e362-e369.

[2] Jeseňák M, Rennerová $Z$, Bánovčin $P$ a kol.: Recidivujúce infekcie dýchacích ciest a imunomodulácia u detí.(Recurrent respiratory tract infections and immunomodulation in children.) 2012, Eds. Aeskulap, Mladá fronta (Czech rep. in Czech language)).

[3] De Benedictis FM, Bush A.: Repeated lower respiratory tract infections in children. BMJ. 2018 July 12; 362: k2698. doi: 10.1136/bmj.k2698.PMID: 30002015.

[4] Pasternak G, Lewandowicz-Uszyńska A, Królak-Olejnik B. [Recurrent respiratory infections in children]. Pol Merkur Lekarski. 2020 Aug 22; 49 (286): 260-266. PMID: 32827422 Review. Polish.

[5] S. Jolles, S. Sánchez-Ramón,. Quinti: Screening protocols for monitoring the respiratory status of primary immunodeficiency disease: findings from the European Working Group on Survey and Subclinical Infections. Respir Res. 2018; 19: 219. 
[6] Pere Soler-Palacín, Javier de Gracia, Luis Ignacio González-Granado, Carlos Martín, Carlos Rodríguez-Gallego, Silvia Sánchez-Ramón, and pulmonary id-group: Primary diseases of immunodeficiency in lung diseases: warning signs, diagnosis and treatment. Respir Res. 2018 November, 12; 19 (1): d219. doi: 10.1186/s12931-018-0923-8. PMID: 30419907.

[7] Bystroň J.: Nemoci z nachlazení z pohledu imunologa. Je potřeba ovlivňovat imunitu $\mathrm{v}$ dětském věku? (Cold diseases from the perspective of an immunologist. Is it necessary to influence immunity in childhood?) Pediatrie pro praxi (Pediatrics for practice). 2019, roč. 20, č. 1, s. 49-54. ISSN: 1213-0494; 1803-5264 (Czech rep.-czech language).

[8] Esposito S, Soto-Martinez ME, Feleszko W, Jones MH, Shen KL, Schaad UB. Non-specific immunomodulators for recurrent respiratory infections, wheezing and asthma in children: a systematic review of mechanistic and clinical evidence. Curr Opin Allergy Clin Immunol. 2018 June; 18 (3): 198-209. doi: 10.1097.

[9] Esposito S, Bianchini S, Bosis S, Tagliabue C, Coro I, Argentiero A, Principi N. A randomized, placebo-controlled, double-blind phase IV study to assess the efficacy and safety of
OM-85 in children with recurrent respiratory infections. J Transl Med. 2019 Aug 23; 17 (1): 284. doi: 10.1186/s12967-019-2040-y. PMID: 31443716.

[10] Yin J, Xu B, Zeng X, Shen K. Broncho-Vaxom in pediatric recurrent respiratory infections: A systematic review and meta-analysis. Int Immunopharmacol. January 2018; 54: 198-209. doi: 10.1016/j.intimp.2017.10.032. Epub 2017 October 16.PMID: 29154122.

[11] Tarantino V, Savaia V, D'Agostino R, Silvestri M, Passali FM, Di Girolamo S, Ciprandi G. Bacteriotherapy in children with recurrent upper respiratory tract infections. Eur Rev Med Pharmacol Sci. 2019 March; 23 (1 Suppl): 39-43. doi: 10.26355/eurrev_201903 17347. PMID: 30920639.

[12] Hawke K, van Driel ML, Buffington BJ, McGuireTM, Homeopathic Remedies for the Prevention and Treatment of Acute Respiratory Infections in Children. Cochrane Syst. 2018 $\begin{array}{lllll}\text { September } & 9 & 9 & \text { (9): } & \text { CD005974. doi: }\end{array}$ 10.1002/14651858.CD005974.pub5.PMID: 30196554. 